\title{
Thyrotoxic Periodic Paralysis: An Update
}

\author{
Jayanta Chakrabortya, c, Semanti Chakraborty ${ }^{a}$, Rita Moitra ${ }^{b}$
}

\begin{abstract}
Thyrotoxic periodic paralysis (TPP) is an endocrine emergency. In initial hours, it requires multidisciplinary care as it involves heart and nervous system. Early diagnosis demands highest clinical acumen as symptoms and signs of thyrotoxicosis are often subtle. TPP is not nowadays confined to particular ethnic groups. Different cellular ion channels have been identified in its etiopathogenesis. Definitive treatment of thyrotoxicosis is also important to prevent relapse. In all cases of hypokalemia in emergency room, TPP should be excluded. Management of hypokalemia should be rationalized to prevent rebound hyperkalemia as total body potassium loss is minimum or nil. Patients should be made euthyroid as soon as possible to prevent relapse of TPP. Future research on ethnic variation of $\mathrm{Na}^{+}-\mathrm{K}^{+}$ATPase activity may enlighten us the cause of higher prevalence in Asians.
\end{abstract}

Keywords: Hypokalemic periodic paralysis; Thyrotoxic periodic paralysis; Graves disease; $\mathrm{Na}^{+}-\mathrm{K}^{+}$ATPase; Kir2.6 channel (KCNJ18)

\section{Introduction}

Thyrotoxic periodic paralysis (TPP) may appear in a patient with thyrotoxicosis due to any cause, such as Graves disease, toxic nodule or thyroiditis. Thyrotoxic symptoms may be very subtle at presentation. A report from California has shown that in more than $50 \%$ of cases of TPP, thyrotoxicosis was not known before [1]. So in all cases of acute hypokalemia with muscle weakness, thyroid functions should be done. Another important development over recent years is that it is now increasingly recognized in non-Asian population also. The patients should be initially managed in intensive care facility as hypokalemia, rebound hyperkalemia and cardiac arrhythmias are all life-threatening and need cardiac

Manuscript submitted June 25, 2020, accepted June 30, 2020

Published online August 26, 2020

aDepartment of Endocrinology and Metabolism, Vivekananda Institute of Medical Sciences, Kolkata, India

bVivekananda Institute of Medical Sciences, Kolkata, India

${ }^{\mathrm{c} C}$ Corresponding Author: Jayanta Chakraborty, Department of Endocrinology and Metabolism, Vivekananda Institute of Medical Sciences, Kolkata, India Email: docjoy4u@gmail.com

doi: https://doi.org/10.14740/jem662 monitoring and urgent pharmaceutical and/or electro-physiologic intervention.

\section{Clinical Feature}

The typical clinical presentation is such that an Asian male, after exercise in the morning or after a heavy carbohydrate meal, sugary drink or alcoholic debauch develop sudden weakness of all four limbs, usually more in lower extremities and unable to stand. Proximal muscles are more involved so unable to comb the hair but able to button the shirts. No breathing problem is noted as respiratory muscles are usually spared. No bowel, bladder involvement is noted. There is irregular heart rate. Arrhythmia may be related to thyrotoxicosis or hypokalemia or both. The cases reported in the literature were almost all between 20 and 40 years. Though there is high prevalence in Asians, there are anecdotal reports of other ethnics too. A single hospital in United States has reported all eight cases in subjects of Hispanic origin [2].

\section{Differential Diagnosis}

TPP should be differentiated from other causes of acute quadriparesis like acute inflammatory demyelinating polyneuropathy (AIDP), botulism, etc. In some cases of TPP, absent deep reflex has been described. But respiratory muscle paralysis common in the above neurological disorders almost always absent in TPP. Other causes of hypokalemia should be differentiated. Initial urine potassium-creatinine ratio helps to differentiate between two broad groups. Those with decreased or normal $\mathrm{K}^{+}$excretion like laxative abuse, gastrointestinal loss, and intracellular shift of $\mathrm{K}^{+}$like TPP, diabetic ketoacidosis, etc., or increased excretion of $\mathrm{K}^{+}$through kidneys due to renal and endocrine diseases. A spot urine potassium to creatinine ratio greater than $13 \mathrm{mEq} / \mathrm{g}$ or $1.5 \mathrm{mEq} / \mathrm{mmol}$ signifies renal $\mathrm{K}^{+}$loss. Following are the causes of increased excretion of $\mathrm{K}^{+}$ in urine. Primary aldosteronism usually presents with hypertension. Plasma aldosterone to plasma renin activity ratio is initial screening test of this disorder. Serum cortisol should be assayed if symptoms suggest to exclude Cushing or ectopic Cushing syndrome. Many forms of congenital adrenal hyperplasia may present with increased mineralocorticoid secretions and hypokalemic hypertension. Liddles syndrome also presents with hypertension. Others, like renal tubular acidosis, Bartter and Gitelman syndrome patients are usually normotensive. Some of these hypokalemic disorders are initially subcat- 
egorized on the basis of acidosis or alkalosis on arterial blood gas analysis which is beyond the scope of this review.

\section{Mechanism and Pathogenesis}

It is more common in Asians, and more so in males as compared to females. Though, Graves disease is more common in females. So genetic and epigenetic factors may be operative. We have presumed five mechanisms may be responsible for this male predominance of TPP. Firstly, androgens may have a stimulating effect on $\mathrm{Na}^{+}-\mathrm{K}^{+}$ATPase activity, though there is no research evidence till date. Secondly, hyper-insulinemia may be more pronounced in males, which stimulates $\mathrm{Na}^{+}-\mathrm{K}^{+}$ATPase activity more in males. Thirdly, there is a possibility of more enhanced response of insulin secretion after carbohydrate-rich meals in males. Fourthly, it is the enhanced sympathetic and catecholamine-induced $\mathrm{Na}^{+}-\mathrm{K}^{+}$ATPase activity in males and lastly due to comparative more muscle mass in males, they may have more $\mathrm{Na}^{+}-\mathrm{K}^{+}$ATPase activity. There may be another defect of outward movement of $\mathrm{K}^{+}$from the cell due to defect of Kir2.6 potassium channel [3]. Loss of function mutation of this channel is found in both sporadic periodic paralysis and TPP [4].

According to evidences available at present that the combined defect of both inward $\mathrm{K}^{+}$channel $\left(\mathrm{Na}^{+}-\mathrm{K}^{+}\right.$ATPase $)$and inward rectifier $\mathrm{K}^{+}$channel (Kir2.6) present in some patients of thyrotoxicosis gives rise to episodes of TPP. The combined gain and loss function mutations in these two channels may be the cause. Gain of function mutation causes hyper-polarization and impaired action potential discharge in Kir2.6 channel (KCNJ18) [5]. The group of patients often present with nomokalemic periodic paralysis. This may be also the reason that TPP is not found in all patients of thyrotoxicosis.

The ethnic variation of TPP has been well recognized but underlying cause is yet to be unveiled. Ethnic variation of $\mathrm{Na}^{+}-$ $\mathrm{K}^{+}$ATPase activity or Kir2.6 inward rectifier channel may be the underlying cause. Though there are chances of other ion channels underlying the disorder. As only up to 33\% of TPP cases have shown Kir2.6 mutations in United States, France and Brazil genetic study [3]. Ethnic variation of red cell $\mathrm{Na}^{+}-$ $\mathrm{K}^{+}$ATPase activity has been seen [6], but no similar study on muscle $\mathrm{Na}^{+}-\mathrm{K}^{+}$ATPase on TTP patients undertaken. So there is scope for future research in this field.

We are sure that further genetic study would tell us the cause of higher susceptibility of Asians for TPP. Moreover male preponderance could be due to any role of androgen receptor or its downstream interactions with muscle ion channels.

\section{Management for Adults}

As total body potassium loss is not there, only intracellular shift of potassium is the cause of hypokalemia. Aggressive potassium replacement often causes rebound hyperkalemia. Potassium chloride replacement should be done with 10 - $20 \mathrm{mmol}$ per liter of intravenous fluid. A published research has shown $24 \mathrm{~h} \mathrm{~K}^{+}$replacement of $90 \mathrm{mEq}$ of potassium chloride or less does not produce rebound hyperkalemia [7]. General opinion of potassium chloride replacement is less than $10 \mathrm{mmol} / \mathrm{hr}$ [8]. However, attending physician is the best judge of how much and how frequently potassium should be given. Treatment should be individualized till there is any guideline available. Frequent monitoring of serum potassium is needed. Propranolol acts by sympathetic blockade. There is less adrenergic stimulation of the enzyme $\mathrm{Na}^{+}-\mathrm{K}^{+}$ATPase, which is responsible for intracellular shift of $\mathrm{K}^{+}$. Propranolol may also reduce sympathetic drive to insulin secretion from beta cells of pancreas. Because we know hyperinsulinemia also stimulate $\mathrm{Na}^{+}-\mathrm{K}^{+}$ATPase activity. Life style modification may help in regaining insulin sensitivity and consequent reduction of $\mathrm{Na}^{+}-\mathrm{K}^{+}$ATPase activity. As TPP can occur in different types of hyperthyroidism, initially antithyroid drugs are started to make the patient euthyroid. Subsequently, it is better to give definitive treatment of radioiodine or thyroid surgery in Graves disease or toxic nodule patients to prevent relapse and consequent episodes of TPP.

\section{Conclusion}

TPP is an endocrine emergency affecting cardiovascular and neurological systems. It is wise to manage it initially in an intensive care unit by a multidisciplinary team. Cardiac monitoring and serum potassium monitoring is of utmost importance in initial few hours. Follow-up treatment of thyrotoxicosis should aim to early reverting to euthyroid state and preventing relapse which may precipitate TPP.

\section{Acknowledgments}

None to declare.

\section{Disclaimer}

This is a review article of literature. It is not a guideline to follow. Authors are not recommending any management protocol. Treating physician should take their decision for management. Authors are not liable for any treatment decisions and consequences.

\section{Financial Disclosure}

None to declare.

\section{Conflict of Interest}

None to declare.

\section{Author Contributions}

Both JC and SC have conceptualized and reviewed the literature. RM has edited the text. 


\section{Data Availability}

The authors declare that data supporting the findings of this study are available within the article.

\section{References}

1. Lauren Hayley Stieger Clarine, Nadeen Hosein. Thyrotoxic periodic paralysis: a review of cases in the last decade. AACE Clinical Case Rep. 2015;1:e182-e186.

2. Ambika Amblee, Swathi Gulati. Thyrotoxic periodic paralysis: eight cases in males of Hispanic origin from a single hospital. AACE Clinical Case Rep. 2016;2:e58e64.

3. Ryan DP, da Silva MR, Soong TW, Fontaine B, Donaldson MR, Kung AW, Jongjaroenprasert W, et al. Mutations in potassium channel Kir2.6 cause susceptibility to thyrotoxic hypokalemic periodic paralysis. Cell.
2010;140(1):88-98.

4. Cheng CJ, Lin SH, Lo YF, Yang SS, Hsu YJ, Cannon $\mathrm{SC}$, Huang CL. Identification and functional characterization of Kir2.6 mutations associated with nonfamilial hypokalemic periodic paralysis. J Biol Chem. 2011;286(31):27425-27435.

5. Soufi M, Ruppert V, Rinne S, Mueller T, Kurt B, Pilz G, Maieron A, et al. Increased KCNJ18 promoter activity as a mechanism in atypical normokalemic periodic paralysis. Neurol Genet. 2018 Oct 3;4(5):e274.

6. Beutler E, Kuhl W, Sacks P. Sodium-potassium-ATPase activity is influenced by ethnic origin and not by obesity. N Engl J Med. 1983;309:756-760.

7. Manoukian MA, Foote JA, Crapo LM. Clinical and metabolic features of thyrotoxic periodic paralysis in 24 episodes. Arch Intern Med. 1999;159(6):601-606.

8. Lu KC, Hsu YJ, Chiu JS, Hsu YD, Lin SH. Effects of potassium supplementation on the recovery of thyrotoxic periodic paralysis. Am J Emerg Med. 2004;22(7):544-547. 